\title{
Blow-up of solutions to a viscoelastic parabolic equation with positive initial energy
}

Haixia $\mathrm{Li}^{1}$ and Yuzhu Han ${ }^{2 *}$

\begin{abstract}
In this paper, a semilinear viscoelastic parabolic equation with nonlinear boundary flux is studied. Due to the comparison principle being invalid, potential well method and concavity argument are used to prove that the solutions blow up in finite time with positive initial energy. This result improves the one obtained by Han et al. (C. R. Math. Acad. Sci. Paris, Sér. I 353:825-830, 2015).
\end{abstract}

Keywords: viscoelastic; potential well method; blow-up; nonlinear boundary flux

\section{Introduction}

In this paper, we are concerned with the blow-up properties of solutions to the semilinear initial boundary value problem of the following form:

$$
\left\{\begin{array}{l}
u_{t}-\Delta u+\int_{0}^{t} g(t-s) \Delta u(x, s) \mathrm{d} s=0, \quad x \in \Omega, 0<t<T, \\
u(x, t)=0, \quad x \in \Gamma_{0}, 0<t<T, \\
\frac{\partial u}{\partial v}-\int_{0}^{t} g(t-s) \frac{\partial u(x, s)}{\partial v} \mathrm{~d} s=|u|^{p-2} u, \quad x \in \Gamma_{1}, 0<t<T, \\
u(x, 0)=u_{0}(x), \quad x \in \Omega,
\end{array}\right.
$$

where $p>2, \Omega \subset \mathbb{R}^{N}(N \geq 2)$ is a bounded domain with smooth boundary $\partial \Omega,\left\{\Gamma_{0}, \Gamma_{1}\right\}$ is a partition of $\partial \Omega$ such that $\partial \Omega=\Gamma_{0} \cup \Gamma_{1}, \Gamma_{0} \cap \Gamma_{1}=\emptyset$ and meas $\left(\Gamma_{0}\right)>0, v$ is the unit outward normal on $\Gamma_{1}$, and the relaxation function $g: \mathbb{R}^{+} \rightarrow \mathbb{R}^{+}$is a bounded $C^{1}$ function satisfying some other conditions to be specified later.

Problems like (1) have their roots in many important physical processes, such as the diffusion of electro-rheological fluids, the heat conduction in materials with memory and viscous flow in viscoelastic materials, a class of materials that have the capacity of dissipation and storage of mechanical energy [2-4]. The main feature of the equation in (1) is that it contains an integro-differential operator, usually called memory term or viscoelastic term, which can be used to represent the damping or memory effect of some special materials. For example, when the heat conduction in materials with memory is investigated, the classical heat flux density $\vec{F}$ is usually replaced by the following form:

$$
\vec{F}=-d \nabla u-\int_{-\infty}^{t} \nabla[k(x, t) u(x, s)] \mathrm{d} s .
$$

(0) The Author(s) 2017. This article is distributed under the terms of the Creative Commons Attribution 4.0 International License (http://creativecommons.org/licenses/by/4.0/), which permits unrestricted use, distribution, and reproduction in any medium, provided you give appropriate credit to the original author(s) and the source, provide a link to the Creative Commons license, and indicate if changes were made. 
Here $u$ denotes the temperature, $d>0$ is the diffusion coefficient and the integral term represents the capacity of the material to keep memory of their past trace. From a mathematical point of view, it is expected that the leading term can dominate the memory term and therefore the equation in (1) is of parabolic type. During the past few years, much work has been done on the study of equations with memory terms, and remarkable progress has been made on the local and global existence, uniqueness, finite time blow-up and regularities of weak or classical solutions. We only refer the interested readers to $[5,6]$ among a large number of literature sources.

In this paper, we confine ourselves to the finite time blow-up property of solutions to Problem (1), an important property possessed by many nonlinear evolution equations. There have been many methods to choose from when determining whether the solutions to the given evolution problem blow up in finite time or not, for instance, the (first) eigenvalue method, the concavity argument, the comparison method based on maximum principle and other methods based on delicate integration. Interested reader may refer to [7] for the outline of each method and their applications to typical examples. Mainly by using the methods mentioned above, blow-up profiles including blow-up time, blow-up rate, blow-up set and boundary layers of solutions to semilinear equations like (1) have been widely investigated when $g(t) \equiv 0$. We only refer to the survey papers $[8,9]$ here.

However, there are much fewer blow-up results for semilinear parabolic equations when $g(t) \not \equiv 0$. When the source term is in the interior of the domain, Messaoudi [10] studied

$$
\left\{\begin{array}{l}
u_{t}-\Delta u+\int_{0}^{t} g(t-s) \Delta u(x, s) \mathrm{d} s=|u|^{p-2} u, \quad x \in \Omega, t>0 \\
u(x, t)=0, \quad x \in \partial \Omega, t>0 \\
u(x, 0)=u_{0}(x), \quad x \in \Omega .
\end{array}\right.
$$

By establishing a connection between $\frac{d}{d t}\|u\|_{2}$ and $\|u\|_{2}^{r}$ with $r>1$, he obtained the finite time blow-up property solutions to (3) when, among other conditions, the initial energy $E(u(0)) \leq 0$, where

$$
E(u(t))=\frac{1}{2}(g \diamond \nabla u)(t)+\frac{1}{2}\left(1-\int_{0}^{t} g(s) \mathrm{d} s\right)\|\nabla u(t)\|_{2}^{2}-\frac{1}{p}\|u(t)\|_{p}^{p},
$$

and

$$
(g \diamond v)(t)=\int_{0}^{t} g(t-s)\|v(x, t)-v(x, s)\|_{2}^{2} \mathrm{~d} s .
$$

This result was later improved by Messaoudi [11] and Fang et al. [12] by showing that the solutions may also blow up in finite time for positive but suitably small initial energy.

When the nonlinearity appears on the boundary, by defining a modified functional and using a concavity argument, Han et al. [1] proved that the solutions to the following problem

$$
\left\{\begin{array}{l}
u_{t}-\Delta u+\int_{0}^{t} g(t-s) \Delta u(x, s) \mathrm{d} s=0, \quad x \in \Omega, t>0, \\
\frac{\partial u}{\partial v}-\int_{0}^{t} g(t-s) \frac{\partial u(x, s)}{\partial v} \mathrm{~d} s=|u|^{p-2} u, \quad x \in \partial \Omega, t>0, \\
u(x, 0)=u_{0}(x), \quad x \in \Omega,
\end{array}\right.
$$

blow up in finite time when the initial energy is strictly negative. 
Compared the blow-up results obtained in $[11,12]$ with the ones in $[1,10]$, it is expected that the solutions to Problem (1) will also blow up in finite time with positive but small initial energy, which is the main purpose of this paper. Since the source is on the boundary, we cannot establish the connection between $\frac{d}{d t}\|u\|_{2}$ and $\|u\|_{2}^{r}$, as was done in [10]. By using the famous potential well method proposed by Sattinger and Payne [13, 14], we will show that when the initial data falls outside of the potential well, the $L^{2}(\Omega)$ norm of the corresponding solution has a positive lower bound, which then can be applied to control the positive initial energy and to derive the finite time blow-up of the corresponding solutions. Similar procedures were used by Marin et al. in dealing with thermoelasticity of micropolar bodies, see $[15,16]$. There are some other interesting works that we have some ideas from, of which we only mention [17-20].

The rest of this paper is organized as follows. In Section 2, as preliminaries, we define some sets and functionals and prove their basic properties. The main result will be stated and proved in Section 3.

\section{Preliminaries}

Let $\Omega, \Gamma_{0}, \Gamma_{1}$ be given as in Section 1 . For any $1 \leq s<\infty$, define

$$
\|u\|_{s}^{s}=\int_{\Omega}|u(x)|^{s} \mathrm{~d} x, \quad\|u\|_{\Gamma_{1}, s}^{s}=\int_{\Gamma_{1}}|u(x)|^{s} \mathrm{~d} \sigma
$$

and the Hilbert space

$$
H \triangleq H_{\Gamma_{0}}^{1}(\Omega)=\left\{u \in H^{1}(\Omega): u=0 \text { on } \Gamma_{0}\right\}
$$

Since $\Gamma_{0}$ has positive $N-1$ dimensional Lebesgue measure, we can equip $H$ with the norm $\|u\|_{H}=\|\nabla u\|_{2}$ that is equivalent to the standard one (see [21]).

Throughout this paper, the relaxation function $g$ and the parameter $p$ are supposed to satisfy

$$
g(s) \geq 0, \quad g^{\prime}(s) \leq 0, \quad 1-\int_{0}^{\infty} g(s) \mathrm{d} s=l>0,
$$

and

$$
2<p \leq \frac{2 N-3}{N-2}, \quad \text { if } N>3 ; \quad p>2, \quad \text { if } N=2 .
$$

Before going further, we present the definition of strong solutions to Problem (1), which was given in [1,22]. Local existence of such a solution was proved in [23] (the first three steps in the proof of Theorem 6) for a little more general problems which contain Problem (1) as a special case, by applying Galerkin's method and the contraction mapping principle. The lengthy proof will not be repeated here.

Definition 2.1 We call $u(x, t)$ a strong solution to Problem (1) if $u \in C([0, T) ; H) \cap$ $C^{1}\left([0, T) ; L^{2}(\Omega)\right)$ and satisfies

$$
\int_{0}^{t} \int_{\Omega}\left(u_{t} \phi+\nabla u \nabla \phi-\int_{0}^{s} g(s-\tau) \nabla u(\tau) \nabla \phi(s) \mathrm{d} \tau\right) \mathrm{d} x \mathrm{~d} s=\int_{0}^{t} \int_{\Gamma_{1}}|u|^{p-2} u \phi \mathrm{d} \sigma \mathrm{d} s
$$

for all $t \in[0, T)$ and all $\phi \in C\left([0, T) ; H^{1}(\Omega)\right)$. 
Assumption (7) is necessary to ensure that the equation in (1) is of parabolic type, and assumption (8) implies $|u|^{p-2} u \in L^{2}\left(\Gamma_{1}\right)$ by the Sobolev trace embedding theorem (Theorem 5.36 in [24]) and hence $\int_{\Gamma_{1}}|u|^{p-2} u \phi \mathrm{d} \sigma$ makes sense.

Let $u(x, t)$ be a strong solution to Problem (1) and define

$$
E(u(t))=\frac{1}{2}(g \diamond \nabla u)(t)+\frac{1}{2}\left(1-\int_{0}^{t} g(s) \mathrm{d} s\right)\|\nabla u(t)\|_{2}^{2}-\frac{1}{p}\|u(t)\|_{\Gamma_{1}, p^{\prime}}^{p}
$$

where $(g \diamond u)(t)$ is given in (5). Then we have the following lemma.

Lemma 2.1 $E(u(t))$ defined in (9) is nonincreasing in $t$ and satisfies

$$
E(u(t))+\int_{0}^{t} \int_{\Omega} u_{t}^{2} \mathrm{~d} x \mathrm{~d} t \leq E\left(u_{0}\right)
$$

Proof Assume first that $u(x, t)$ is sufficiently smooth. Multiplying the equation in (1) by $u_{t}$ and integrating by parts over $\Omega$ yield

$$
\begin{aligned}
\int_{\Omega} u_{t}^{2} \mathrm{~d} x= & \int_{\Omega} \Delta u u_{t} \mathrm{~d} x-\int_{\Omega} u_{t}\left(\int_{0}^{t} g(t-s) \Delta u(x, s) \mathrm{d} s\right) \mathrm{d} x \\
= & \int_{\Gamma_{1}} u_{t}\left(\frac{\partial u}{\partial v}-\int_{0}^{t} g(t-s) \frac{\partial u}{\partial v} \mathrm{~d} s\right) \mathrm{d} \sigma-\int_{\Omega} \nabla u \nabla u_{t} \mathrm{~d} x \\
& +\int_{\Omega}\left(\int_{0}^{t} g(t-s) \nabla u(s) \nabla u_{t}(t) \mathrm{d} s\right) \mathrm{d} x \\
= & -\frac{\mathrm{d}}{\mathrm{d} t}\left(\frac{1}{2} \int_{\Omega}|\nabla u|^{2} \mathrm{~d} x\right)+\frac{\mathrm{d}}{\mathrm{d} t}\left(\frac{1}{p} \int_{\Gamma_{1}}|u|^{p} \mathrm{~d} \sigma\right) \\
& +\int_{0}^{t} g(t-s) \int_{\Omega} \nabla u(s) \nabla u_{t}(t) \mathrm{d} x \mathrm{~d} s .
\end{aligned}
$$

Rewrite the last term of the right-hand side of the above equality as follows:

$$
\begin{aligned}
& \int_{0}^{t} g(t-s) \int_{\Omega} \nabla u(s) \nabla u_{t}(t) \mathrm{d} x \mathrm{~d} s \\
&=- \frac{1}{2} \frac{\mathrm{d}}{\mathrm{d} t}\left(\int_{0}^{t} g(t-s) \int_{\Omega}|\nabla u(s)-\nabla u(t)|^{2} \mathrm{~d} x \mathrm{~d} s\right)+\frac{1}{2} \frac{\mathrm{d}}{\mathrm{d} t}\left(\int_{0}^{t} g(s) \mathrm{d} s \int_{\Omega}|\nabla u(t)|^{2} \mathrm{~d} x\right) \\
& \quad+\frac{1}{2} \int_{0}^{t} g^{\prime}(t-s) \int_{\Omega}|\nabla u(s)-\nabla u(t)|^{2} \mathrm{~d} x \mathrm{~d} s-\frac{1}{2} g(t) \int_{\Omega}|\nabla u(t)|^{2} \mathrm{~d} x
\end{aligned}
$$

Substituting (12) into (11) and recalling (7) and (9), we see that

$$
\begin{aligned}
\frac{\mathrm{d}}{\mathrm{d} t} E(u(t))= & -\int_{\Omega} u_{t}^{2} \mathrm{~d} x-\frac{1}{2} g(t) \int_{\Omega}|\nabla u(t)|^{2} \mathrm{~d} x \\
& +\frac{1}{2} \int_{0}^{t} g^{\prime}(t-s) \int_{\Omega}|\nabla u(s)-\nabla u(t)|^{2} \mathrm{~d} x \mathrm{~d} s \\
\leq & -\int_{\Omega} u_{t}^{2} \mathrm{~d} x \leq 0 .
\end{aligned}
$$


Therefore, the conclusion of this lemma is true for smooth solution $u(x, t)$. By a standard density argument, it is known that the same result can be established for strong solutions and for almost every $t$. The proof is complete.

Next, let $l$ be the constant given in (7). For any $u \in H$, set

$$
\begin{aligned}
& J(u)=\frac{l}{2}\|\nabla u\|_{2}^{2}-\frac{1}{p}\|u\|_{\Gamma_{1}, p}^{p}, \\
& I(u)=l\|\nabla u\|_{2}^{2}-\|u\|_{\Gamma_{1}, p}^{p}, \\
& \mathcal{N}=\left\{u \in H: I(u)=0,\|\nabla u\|_{2} \neq 0\right\} .
\end{aligned}
$$

We then define the potential well and its corresponding set as follows:

$$
\begin{aligned}
& W=\{u \in H: I(u)>0, J(u)<d\} \cup\{0\}, \\
& V=\{u \in H: I(u)<0, J(u)<d\},
\end{aligned}
$$

where the depth $d$ of the potential well $W$ is characterized as

$$
d=\inf _{u \in H \backslash\{0\}}\left\{\sup _{\lambda>0} J(\lambda u)\right\}=\inf _{u \in \mathcal{N}} J(u) .
$$

The positivity of $d$ is given in the next lemma.

Lemma 2.2 The depth $d$ of the potential well $W$ is positive.

Proof Since $p$ satisfies (8), $H$ can be embedded into $L^{p}\left(\Gamma_{1}\right)$ continuously. Let $S>0$ be the best embedding constant, i.e., $\|u\|_{\Gamma_{1}, p} \leq S\|\nabla u\|_{2}, \forall u \in H$.

For any $u \in \mathcal{N}$, we have

$$
l\|\nabla u\|_{2}^{2}=\|u\|_{\Gamma_{1}, p}^{p} \leq S^{p}\|\nabla u\|_{2}^{p},
$$

which implies $\|\nabla u\|_{2} \geq\left(\frac{l}{S^{p}}\right)^{\frac{1}{p-2}}$. Therefore, for any $u \in \mathcal{N}$,

$$
J(u)=\frac{l}{2}\|\nabla u\|_{2}^{2}-\frac{1}{p}\|u\|_{\Gamma_{1}, p}^{p}=\left(\frac{1}{2}-\frac{1}{p}\right) l\|\nabla u\|_{2}^{2} \geq \frac{(p-2) l}{2 p}\left(\frac{l}{S^{p}}\right)^{\frac{2}{p-2}}>0 .
$$

By the definition of $d$, it is seen that $d>0$. The proof is complete.

The next lemma describes the invariance of $V$ with respect to the semiflow of (1) under some additional conditions.

Lemma 2.3 Let (7) and (8) hold and $u(x, t)$ be a local solution to Problem (1). If there exists $t_{0} \in[0, T)$ such that $u\left(\cdot, t_{0}\right) \in V$ and $E\left(u\left(t_{0}\right)\right)<d$, then $u(x, t)$ remains inside $V$ for any $t \in\left[t_{0}, T\right)$, where $T$ is the maximal existence time of $u(x, t)$.

Proof Suppose on the contrary that there exists $t_{1} \in[0, T)$ such that $u(x, t) \in V$ for $t \in$ $\left[t_{0}, t_{1}\right)$ and $u\left(x, t_{1}\right) \notin V$. By the definition of $V$ and the continuity of $J(u(x, t))$ and $I(u(x, t))$ with respect to $t$, we have either (i) $J\left(u\left(x, t_{1}\right)\right)=d$ or (ii) $I\left(u\left(x, t_{1}\right)\right)=0$. 
By Lemma 2.1 we know that $E\left(u\left(t_{1}\right)\right) \leq E\left(u\left(t_{0}\right)\right)$, which implies

$$
J\left(u\left(x, t_{1}\right)\right) \leq E\left(u\left(t_{1}\right)\right) \leq E\left(u\left(t_{0}\right)\right)<d
$$

So case (i) is impossible.

To prove that case (ii) is also impossible, we first show that there is a positive constant $c^{*}$ such that for any $u \in V,\|\nabla u\|_{2} \geq c^{*}$. Indeed, for any $u \in V$, it holds that

$$
l\|\nabla u\|_{2}^{2}<\|u\|_{\Gamma_{1}, p}^{p} \leq S^{p}\|\nabla u\|_{2}^{p}
$$

which shows $\|\nabla u\|_{2}>\left(\frac{l}{S^{p}}\right)^{\frac{1}{p-2}} \triangleq c^{*}$. Here $S>0$ is the embedding constant given in Lemma 2.2.

Since $u(x, t) \in V$ for $t \in\left[t_{0}, t_{1}\right)$, we have $\|\nabla u(\cdot, t)\|_{2} \geq c^{*}$ for all $t \in\left[t_{0}, t_{1}\right)$. By continuity, it also holds that $\left\|\nabla u\left(\cdot, t_{1}\right)\right\|_{2} \geq c^{*}$. This together with (ii) implies that $u\left(x, t_{1}\right) \in \mathcal{N}$. By the definition of $d$, we have $J\left(u\left(x, t_{1}\right)\right) \geq d$, a contradiction. The proof is complete.

\section{Main result}

The main result of this paper is the following.

Theorem 3.1 Suppose that (7), (8) hold and that $u_{0} \in V$ satisfies $E\left(u_{0}\right)<\frac{1-l}{2}\left(\frac{l}{S^{p}}\right)^{\frac{2}{p-2}}$, where $l$ and $S$ are the positive constants defined in (7) and Lemma 2.2, respectively. If

$$
\int_{0}^{\infty} g(s) \mathrm{d} s<\frac{p-2}{p-3 / 2}
$$

then any strong solution $u(x, t)$ to Problem (1) blows up in finite time $T$ in the sense that $\lim _{t \rightarrow T}\|u(\cdot, t)\|_{2}=+\infty$.

Proof We will prove this theorem by combining Lemma 2.3 with the concavity argument introduced by Levine. Suppose on the contrary that the solution $u$ is global, then

$$
M(t)=\int_{0}^{t} \int_{\Omega} u^{2}(x, \tau) \mathrm{d} x \mathrm{~d} \tau+A
$$

is well defined for all $t>0$, where $A>0$ is a constant to be determined. Direct computations show that

$$
M^{\prime}(t)=\int_{\Omega} u^{2}(x, t) \mathrm{d} x
$$

and

$$
\begin{aligned}
M^{\prime \prime}(t)=2 \int_{\Omega} u u_{t} \mathrm{~d} x= & 2 \int_{\Gamma_{1}}|u|^{p} \mathrm{~d} \sigma-2\left(1-\int_{0}^{t} g(s) \mathrm{d} s\right) \int_{\Omega}|\nabla u|^{2} \mathrm{~d} x \\
& +2 \int_{0}^{t} g(t-s) \int_{\Omega} \nabla u(t)(\nabla u(s)-\nabla u(t)) \mathrm{d} x \mathrm{~d} s
\end{aligned}
$$


By applying the Cauchy-Schwarz inequality first and Young's inequality then to the third term on the right-hand side of (17), we obtain

$$
\begin{aligned}
M^{\prime \prime}(t) \geq 2 & \int_{\Gamma_{1}}|u|^{p} \mathrm{~d} \sigma-2\left(1-\int_{0}^{t} g(s) \mathrm{d} s\right) \int_{\Omega}|\nabla u|^{2} \mathrm{~d} x \\
& -2 \int_{0}^{t} g(t-s)\|\nabla u(t)\|_{2}\|\nabla u(t)-\nabla u(s)\|_{2} \mathrm{~d} s \\
\geq 2 & {\left[\int_{\Gamma_{1}}|u|^{p} \mathrm{~d} \sigma-\left(1-\frac{3}{4} \int_{0}^{t} g(s) \mathrm{d} s\right) \int_{\Omega}|\nabla u|^{2} \mathrm{~d} x-(g \diamond \nabla u)(t)\right] . }
\end{aligned}
$$

It then follows from (7), (9), (15) and (18) that there exists a constant $\delta>0$ such that

$$
\begin{aligned}
M^{\prime \prime}(t) \geq & -4(1+\delta) E(u(t)) \\
= & 4(1+\delta)\left(-E\left(u_{0}\right)+\int_{0}^{t} \int_{\Omega} u_{\tau}^{2} \mathrm{~d} x \mathrm{~d} \tau+\frac{1}{2} \int_{0}^{t} g(\tau)\|\nabla u(\tau)\|_{2}^{2} \mathrm{~d} \tau\right. \\
& \left.-\frac{1}{2} \int_{0}^{t} \int_{0}^{\tau} g^{\prime}(\tau-s) \int_{\Omega}|\nabla u(s)-\nabla u(\tau)|^{2} \mathrm{~d} x \mathrm{~d} s \mathrm{~d} \tau\right) \\
\geq & 4(1+\delta)\left(-E\left(u_{0}\right)+\int_{0}^{t} \int_{\Omega} u_{\tau}^{2} \mathrm{~d} x \mathrm{~d} \tau+\frac{1}{2} \int_{0}^{t} g(\tau)\|\nabla u(\tau)\|_{2}^{2} \mathrm{~d} \tau\right) .
\end{aligned}
$$

On the other hand,

$$
M^{\prime}(t)=\int_{\Omega} u^{2}(x, t) \mathrm{d} x=2 \int_{0}^{t} \int_{\Omega} u u_{\tau} \mathrm{d} x \mathrm{~d} \tau+\int_{\Omega} u_{0}^{2}(x) \mathrm{d} x
$$

which yields, with the help of Cauchy's inequality with $\varepsilon>0$, that

$$
M^{\prime}(t)^{2} \leq 4(1+\varepsilon) \int_{0}^{t} \int_{\Omega} u^{2} \mathrm{~d} x \mathrm{~d} \tau \int_{0}^{t} \int_{\Omega} u_{\tau}^{2} \mathrm{~d} x \mathrm{~d} \tau+\left(1+\frac{1}{\varepsilon}\right)\left(\int_{\Omega} u_{0}^{2}(x) \mathrm{d} x\right)^{2}
$$

Combining (19) with (20) and recalling (16), we obtain, for any $\alpha>0$, that

$$
\begin{aligned}
& M^{\prime \prime}(t) M(t)-(1+\alpha) M^{\prime}(t)^{2} \\
& \geq 4(1+\delta)\left(-E\left(u_{0}\right)+\int_{0}^{t} \int_{\Omega} u_{\tau}^{2} \mathrm{~d} x \mathrm{~d} \tau+\frac{1}{2} \int_{0}^{t} g(\tau)\|\nabla u(\tau)\|_{2}^{2} \mathrm{~d} \tau\right) \\
& \quad \times\left(\int_{0}^{t} \int_{\Omega} u^{2}(x, \tau) \mathrm{d} x \mathrm{~d} \tau+A\right) \\
& \quad-4(1+\alpha)(1+\varepsilon) \int_{0}^{t} \int_{\Omega} u^{2} \mathrm{~d} x \mathrm{~d} \tau \int_{0}^{t} \int_{\Omega} u_{\tau}^{2} \mathrm{~d} x \mathrm{~d} \tau \\
& \quad-(1+\alpha)\left(1+\frac{1}{\varepsilon}\right)\left(\int_{\Omega} u_{0}^{2}(x) \mathrm{d} x\right)^{2} .
\end{aligned}
$$

Fix $\varepsilon$ and $\alpha$ small enough such that

$$
1+\delta>(1+\alpha)(1+\varepsilon) .
$$


By Lemma 2.3 we know $\|\nabla u(\cdot, t)\| \geq\left(\frac{l}{S^{p}}\right)^{\frac{1}{p-2}}$ for all $t \geq 0$. From $\lim _{t \rightarrow+\infty} \int_{0}^{t} g(\tau) \mathrm{d} \tau=1-l$ and $E\left(u_{0}\right)<\frac{1-l}{2}\left(\frac{l}{S^{p}}\right)^{\frac{2}{p-2}}$ it can be deduced that there exists $t^{*}>0$ such that

$$
\frac{1}{2} \int_{0}^{t} g(\tau)\|\nabla u(\tau)\|_{2}^{2} \mathrm{~d} \tau>E\left(u_{0}\right), \quad \forall t \geq t^{*}
$$

It then follows from (21), (22) and (23) that

$$
M^{\prime \prime}(t) M(t)-(1+\alpha) M^{\prime}(t)^{2}>0, \quad \forall t \geq t^{*}
$$

for $A>0$ suitably large, which implies

$$
\frac{\mathrm{d}}{\mathrm{d} t}\left(\frac{M^{\prime}(t)}{M^{1+\alpha}(t)}\right)>0, \quad \forall t \geq t^{*}
$$

or

$$
\frac{M^{\prime}(t)}{M^{1+\alpha}(t)}>\frac{M^{\prime}\left(t^{*}\right)}{M^{1+\alpha}\left(t^{*}\right)}, \quad \forall t>t^{*}
$$

Integrating both sides of $(25)$ over $\left(t^{*}, t\right)$, we can see that $M(t)$ cannot remain finite for all $t>t^{*}$, and therefore reaches a contradiction. The proof is complete.

\section{Acknowledgements}

The authors would like to thank the referees for their valuable comments and suggestions regarding the original manuscript and for pointing out several references that are quite helpful to us. They would also like to express their sincere gratitude to Professor Wenjie Gao for his enthusiastic guidance and constant encouragement. The first author is supported by NSFC (11626044) and by the Natural Science Foundation of Changchun Normal University. The second author is supported by NSFC (11401252) and by Science and Technology Development Project of Jilin Province (20160520103JH).

\section{Competing interests}

The authors declare that they have no competing interests.

\section{Authors' contributions}

All authors contributed equally to the manuscript and read and approved the final manuscript.

\section{Author details}

'School of Mathematics, Changchun Normal University, Changchun, 130032, P.R. China. ${ }^{2}$ School of Mathematics, Jilin University, No. 2699 Qianjin Street, Changchun, 130012, P.R. China.

\section{Publisher's Note}

Springer Nature remains neutral with regard to jurisdictional claims in published maps and institutional affiliations.

Received: 18 March 2017 Accepted: 30 May 2017 Published online: 08 June 2017

\section{References}

1. Han, YZ, Gao, WJ, Li, HX: Blow-up of solutions to a semilinear heat equation with a viscoelastic term and a nonlinear boundary flux. C. R. Math. Acad. Sci. Paris, Sér. I 353(9), 825-830 (2015)

2. Acerbi, E, Mingione, G: Regularity results for stationary electrorheological fluids. Arch. Ration. Mech. Anal. 164, 213-259 (2002)

3. Cavalcanti, M, Domingos Cavalcanti, V, Lasiecka, I, Webler, C: Intrinsic decay rates for the energy of a nonlinear viscoelastic equation modeling the vibrations of thin rods with variable density. Adv. Nonlinear Anal. 6(2), 121-145 (2017)

4. Ruzicka, M: Electrorheological Fluids: Modelling and Mathematical Theory. Lecture Notes in Math., vol. 1748. Springer, Berlin (2000)

5. Yin, HM: On parabolic Volterra equations in several space dimensions. SIAM J. Math. Anal. 22, 1723-1737 (1991)

6. Yin, HM: Weak and classical solutions of some Volterra integro-differential equations. Commun. Partial Differ. Equ. 17, 1369-1385 (1992)

7. Hu, B: Blow-up Theories for Semilinear Parabolic Equations. Springer, Berlin (2011) 
8. Galaktionov, VA, Vázquez, JL: The problem of blow-up in nonlinear parabolic equations. Discrete Contin. Dyn. Syst. 8(2), 399-434 (2002)

9. Levine, HA: The role of critical exponents in blow-up problems. SIAM Rev. 32, 262-288 (1990)

10. Messaoudi, SA: Blow-up of solutions of a semilinear heat equation with a visco-elastic term. Prog. Nonlinear Differ. Equ. Appl. 64, 351-356 (2005)

11. Messaoudi, SA: Blow up of solutions of a semilinear heat equation with a memory term. Abstr. Appl. Anal. 2, 87-94 (2005)

12. Fang, ZB, Sun, L: Blow up of solutions with positive initial energy for the nonlocal semilinear heat equation. J. Korean Soc. Ind. Appl. Math. 16, 235-242 (2012)

13. Sattinger, DH: On global solution of nonlinear hyperbolic equations. Arch. Ration. Mech. Anal. 30(2), 148-172 (1968)

14. Payne, LE, Sattinger, DH: Saddle points and instability of nonlinear hyperbolic equations. Isr. J. Math. 22, 273-303 (1975)

15. Marin, M, Craciun, EM, Pop, N: Consideration on mixed initial-boundary value problems for micropolar porous bodies Dyn. Syst. Appl. 25, 175-196 (2016)

16. Marin, M, Lupu, M: On harmonic vibrations in thermoelasticity of micropolar bodies. J. Vib. Control 4(5), 507-518 (1998)

17. Ahmad, S, Le, D: Global and blow up solutions to cross diffusion systems. Adv. Nonlinear Anal. 4(3), 209-219 (2015)

18. Bougherara, B, Giacomoni, J: Existence of mild solutions for a singular parabolic equation and stabilization. Adv. Nonlinear Anal. 4(2), 123-134 (2015)

19. Radulescu, V: Nonlinear elliptic equations with variable exponent: old and new. Nonlinear Anal. 121, 336-369 (2015)

20. Radulescu, V, Repovs, D: Partial Differential Equations with Variable Exponents. Variational Methods and Qualitative Analysis. Monographs and Research Notes in Mathematics. CRC Press, Boca Raton (2015)

21. Levine, HA, Smith, RA: A potential well theory for the wave equation with a nonlinear boundary condition. J. Reine Angew. Math. 374, 1-23 (1987)

22. Pucci, P, Serrin, J: Asymptotic stability for nonlinear parabolic systems. In: Energy Methods in Continuum Mechanics, pp. 66-74. Springer, Berlin (1996)

23. Fang, ZB, Qiu, LR: Global existence and uniform energy decay rates for the semilinear parabolic equation with a memory term and mixed boundary condition. Abstr. Appl. Anal. 2013, 1 (2013)

24. Adams, RA, Fournier, JJF: Sobolev Spaces. Elsevier, Amsterdam (2003)

\section{Submit your manuscript to a SpringerOpen ${ }^{\ominus}$ journal and benefit from:}

- Convenient online submission

Rigorous peer review

Immediate publication on acceptance

- Open access: articles freely available online

- High visibility within the field

- Retaining the copyright to your article 